\title{
Percutaneous transhepatic biliary drainage may be the preferred preoperative drainage method in hilar cholangio- carcinoma
}

\section{다)(1) $(5)$}

\section{Authors}

Yongjiang Ba1, 2,3, " , Ping Yue 1,2,4,5,6, ", Joseph W. Leung7, Haiping Wang1,5,6, Yanyan Lin 1, 2,4,5,6, Bing Bai ${ }^{1,2,4,5,6}$, Xiaoliang Zhu ${ }^{1,4,5,6,9}$, Lei Zhang ${ }^{1,4,5,6,8}$, Kexiang Zhu' ${ }^{1,4,5,6,9}$, Wenhui Wang ${ }^{1,10}$, Wenbo Meng ${ }^{1,2,4,5,6}$, Wence Zhou ${ }^{1,4,5,6,9}$, Ying Liu' ${ }^{11}$, Xun Li ${ }^{1,4,5,6,8}$

Institutions

1 The First Clinical Medical School of Lanzhou University, Lanzhou, China

2 Department of Special Minimally Invasive Surgery, The First Hospital of Lanzhou University, Lanzhou, China

3 The Second Department of General Surgery, The First People's Hospital of Qujing City, Qujing, China

4 Hepatopancreatobiliary Surgery Institute of Gansu Province, Lanzhou, Gansu, China

5 Key Laboratory of Biological Therapy And Regenerative Medicine Transformation Gansu Province, Lanzhou, China

6 Clinical Medical College Cancer Center of Lanzhou University, Lanzhou, China

7 Division of Gastroenterology and Hepatology, UC Davis Medical Center, and Section of Gastroenterology, Sacramento VA Medical Center, Sacramento, California, United States

8 The Fifth Department of General Surgery, The First Hospital of Lanzhou University, Lanzhou, China

9 The Second Department of General Surgery, The First Hospital of Lanzhou University, Lanzhou, China

10 The Department of Interventional Medicine, The First Hospital of Lanzhou University, Lanzhou, China

11 Foreign Languages Department of Lanzhou University, Lanzhou, China

submitted 8.1.2019

accepted after revision 22.5.2019

Bibliography

DOI https://doi.org/10.1055/a-0990-9114 |

Endoscopy International Open 2020; 08: E203-E210

(c) Georg Thieme Verlag KG Stuttgart · New York eISSN 2196-9736
Corresponding author

Wenbo Meng, The First Hospital of Lanzhou University, Department of Special Minimally Invasive Surgery, NO.1 DongGang West Road, LanZhou, Gansu 730000, Lanzhou 730000, China

Fax: +869318356022

mengwb@Izu.edu.cn

\section{ABSTRACT}

Background and study aims Preoperative biliary drainage of hilar cholangiocarcinoma (HC) is controversial. The goal of this study was to compare the clinical outcome and associated complications for types II, III, and IV HC managed by percutaneous transhepatic biliary drainage (PTBD) and endoscopic retrograde cholangiopancreatography (ERCP).

Patients and methods Between January 2011 and June 2017, a total of 180 patients with II, III, and IV HC were enrolled in this retrospective cohort study. According to the drainage method, patients were divided into two groups: PTBD $(n=81)$ and ERCP $(n=99)$. This study was registered with ClinicalTrials.gov, NCT03104582, and was completed. Results Compared with the PTBD group, the ERCP group had a higher incidence of post-procedural cholangitis (37 [37.37\%] vs. 18 [22.22\%], $P=0.028)$ and pancreatitis (17 [17.17\%] vs. 2 [2.47\%], $P=0.001)$; required more salvaged biliary drainage (18 [18.18\%] vs. 5 [6.17\%], $P=0.029)$, and incurred a higher cost $(P<0.05)$. Patients with type III and IV $\mathrm{HC}$ in the ERCP group had more cholangitis than those in the PTBD group (26 [36.62\%] vs. 11 [18.03\%], $P=0.018)$. The rate of cholangitis in patients who received endoscopic bilateral biliary stents insertion was higher than patients with unilateral stenting $(23$ [50.00\%] vs. 9 [26.47\%], $P=$ 0.034 ), and underwent PTBD internal-external drainage had a higher incidence of cholangitis than those with only external drainage (11 [34.36\%] vs. 7 [14.29\%], $P=0.034$ ). No significant difference in the rate of cholangitis was observed between the endoscopic unilateral stenting

* Yongjiang Ba and Ping Yue make the same contribution to this work. 
group and the endoscopic nasobiliary drainage group (9 [26.47\%] vs. 5 [26.32\%], $P=0.990)$.

Conclusion Compared to ERCP, PTBD reduced the rate of cholangitis, pancreatitis, salvage biliary drainage, and de- creased hospitalization costs in patients with types II, III, and IV HC. Risk of cholangitis for patients with types III and IV was significantly lower in the PTBD group.

\section{Introduction}

Hilar cholangiocarcinoma (HC, or Klatskin tumor) is considered highly malignant due to its aggressive nature with early invasion into the adjacent liver and thus a poor prognosis. It accounts for $40 \%$ to $60 \%$ of all extrahepatic bile duct carcinomas [1]. Currently, radical resection is still considered one of the most effective treatments, especially for Bismuth-Corlette type II, III, and IV lesions. Due to the slow-growing nature of the tumor, most lesions are found in an advanced stage when patients present with obstructive jaundice, involvement of the segmental bile ducts, and invasion into the liver, which makes complete resection difficult and, thus, radical resection rates are low [2-5]. Tumor involvement of the liver hilum leads to extensive intrahepatic bile duct obstruction, resulting in damage to the liver, nervous system, and cardiovascular and urinary systems. Tumor invasion could involve the cystic duct and extension to the intrahepatic bile ducts. Resection of the hilar tumor with extended hepatectomy offers the best chance for cure. Although $\mathrm{HC}$ can be diagnosed at an early stage, the current reported R0 resection rate is less than $30 \%$, and a reported 5year postoperative survival rate ranges from $30 \%$ to $40 \%[6,7]$.

Current consensus indicates that preoperative biliary drainage is beneficial to improve postoperative liver function [8]. Endoscopic retrograde cholangiopancreatography (ERCP) and percutaneous transhepatic biliary drainage (PTBD) are frequently used to provide biliary drainage because both methods are effective in relieving the obstructive jaundice. However, these invasive procedures are associated with an increase in postprocedural complications, including cholangitis, pancreatitis, and hemobilia. Recent publications have suggested that cholangitis could negatively affect clinical outcome and prognosis. So far it is still unclear whether ERCP or PTBD is the preferred preoperative drainage method. Our study aimed to investigate the optimal preoperative biliary drainage methods to prevent post-procedural cholangitis in type II, III, and IV hilar cholangiocarcinoma.

\section{Patients and methods}

\section{Study design and patients}

Between January 2011 and June 2017, 339 patients with hilar bile duct stricture who required bile drainage were recruited into the study. Diagnosis of $\mathrm{HC}$ depends on results of its clinical manifestation, serum biomarkers and imaging examination. Patients with malnutrition, hypoalbuminemia, suspected cholangitis, long-term jaundice, planned preoperative anti-neoplastic therapy and future liver remnant $(F L R)<30 \%$ were considered to need preoperative bile drainage. One hundred fifty- nine patients were excluded because of type I HC, hepatic duct stones with stenosis, gallbladder carcinoma, metastatic tumors of hilar, primary sclerosing cholangitis (PSC), IgG4-Related sclerosing cholangitis (IgG4-SC), hepatic echinococcosis, inability to undergo percutaneous biliary drainage, or failure of ERCP or placement of a percutaneous transhepatic cholangiography. Eventually, 180 eligible patients with type II, III, and IV hilar cholangiocarcinoma were included in this study ( $>$ Fig. 1). According to the drainage method, the patients were divided into two groups: ERCP group and PTBD group. The ERCP group included patients who underwent endoscopic biliary stenting (EBS) and endoscopic nasobiliary drainage (ENBD).

\section{Drainage method}

\section{ERCP}

When performing ERCP, bile duct cannulation and cholangiogram procedures were completed by using a duodenoscope (TJF-260V, Olympus, Japan). In a general way, the guide wire was inserted and crossed over the malignant obstruction site to the proximal end of the hepatic bile duct. Considering that patients may undergo radical cholangiocarcinoma resection

339 patients with peri hilar bile duct stricture

140 patients were excluded

65 HC type I

37 hepatic duct stones with stenosis

15 gallbladder carcinoma

11 metastatic tumors of hilar

8 primary sclerosing cholangitis (PSC)

2 IgG4-related sclerosing cholangitis (IgG4-SC)

2 hepatic echinococcosis

199 HC type II, III and IV patients confirmed

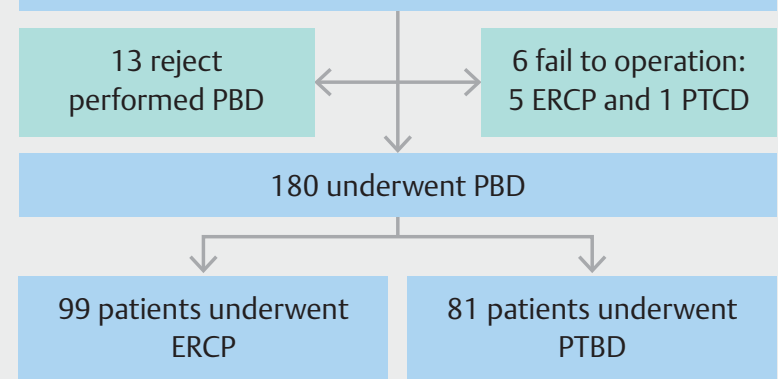

Fig. 1 Flow diagram of the study. 

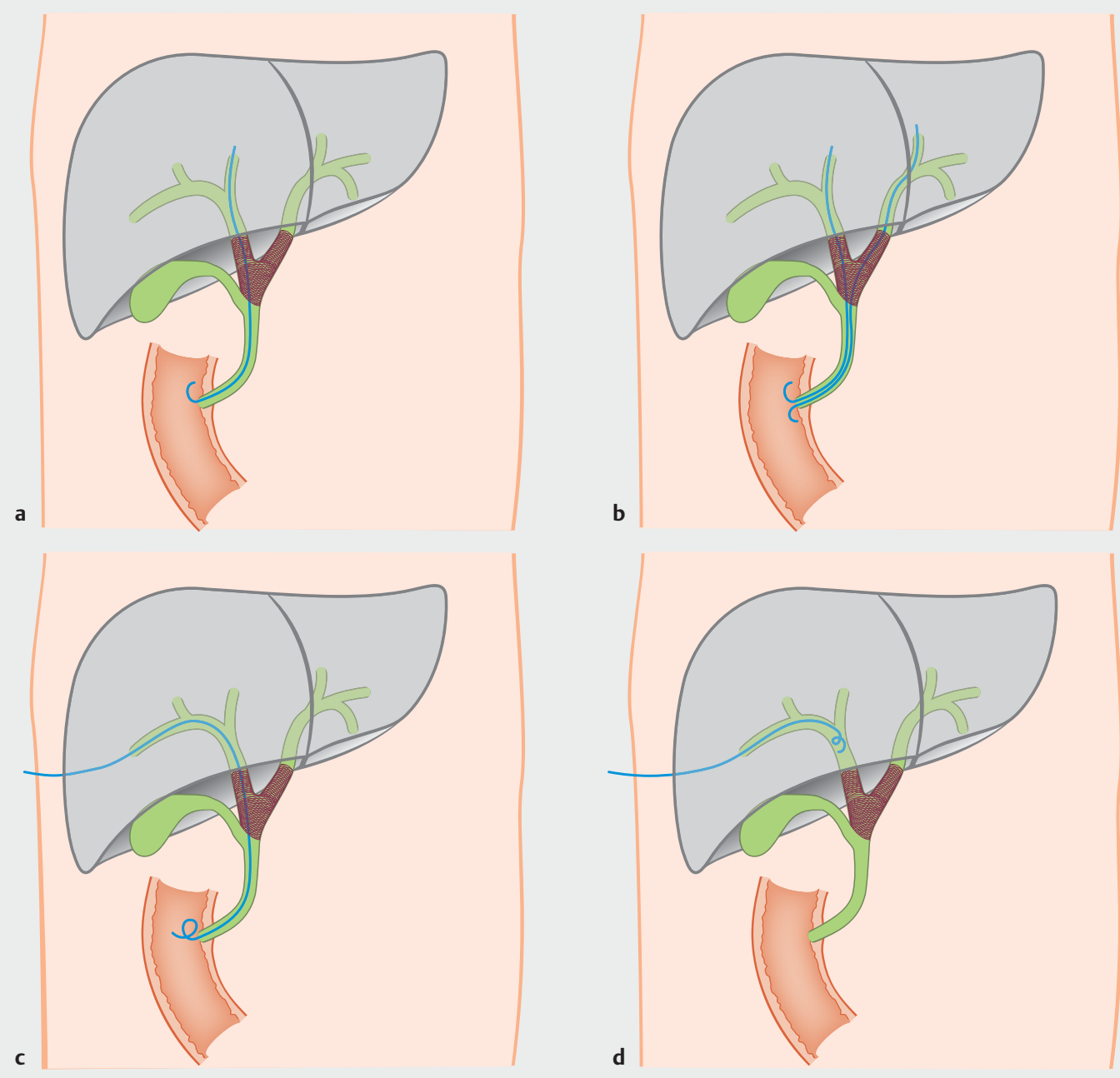

> Fig. 2 Various biliary drainage methods. a Unilateral EBS. b Bilateral EBS. c Internal-external PTBD. d External PTBD.

when jaundice reduction is satisfactory, one or two plastic biliary stents or endoscopic nasobiliary drains were placed (8.5 Fr; Boston Scientific, United States or Olympus, Japan). If the guide wire first entered the bile duct on the side of the future remnant lobe, a unilateral plastic stent or ENBD was usually placed; if the guide wire entered the bile duct of the non-future remnant lobe first and then the bile duct of the future remnant lobe was selected, bilateral biliary stents were placed side by side ( $\vee$ Fig. 2a, $\triangleright$ Fig.2b). Generally speaking, ENBD was performed when patients had difficulty during contrast agent excretion, or when bile was thick or full of dregs, which is easy to clog drainage tubes. The upper edge of plastic stents and the tip of the nasobiliary catheter ( $8 \mathrm{Fr} / 10.2 \mathrm{Fr}$; Cook Medical, Winston-Salem, North Carolina, United States) should cross over the upper margin of the malignant obstruction site by $2 \mathrm{~cm}$.

\section{PTBD}

The dilated intrahepatic bile duct of the expected retention liver was selected based on imaging findings. Ultrasound or x-rayguided percutaneous transhepatic cholangiography was achieved by using a trocar to penetrate the target intrahepatic bile duct. The guidewire was then advanced through the trocar to lead the tube insertion. After the guidewire passed over the malignant stenosis site and the duodenal papilla, a catheter (7Fr; Olympus, Japan or Cook Medical, Winston-Salem, North Carolina, United States) was placed with its distal end along the guidewire in the duodenum for internal-external drainage. PTBD external drainage was performed if the guidewire failed to pass the malignant stenosis site of the intrahepatic bile $\operatorname{duct}(\triangleright$ Fig. 2c, $\triangleright$ Fig. $2 \mathrm{~d}$ ).

\section{Data collection}

Patient demographics, including age and sex, and biochemistry, including tumor marker CA19-9, white blood cell (WBC) count, serum amylase level, and electrolytes were collected before the drainage procedure. Total bilirubin (TBIL) level, WBC, serum amylase, and electrolyte levels were checked again at 24 and 48 hours and 1 and 2 weeks after biliary drainage. Data on body temperature, color of bile, and abdominal pain score (evaluated by numerical rating scale [NRS]) were also recorded. 
Complications, including acute cholangitis, pancreatitis, and hemobilia, were recorded [9]. Diagnostic criteria for acute cholangitis were based on the TG13 Tokyo Guidelines and included: (1) upper abdominal pain (NRS $\geq 4)$; (2) fever $\left(>38.0^{\circ} \mathrm{C}\right.$ ) and/or chills; (3) serum total bilirubin (TBIL) level $>34.2 \mu \mathrm{mol} / \mathrm{L}$; and (4) inflammatory response (WBC $>10,000 / \mu \mathrm{L}, C R P>1 \mathrm{mg} / \mathrm{dL}$ ) [10]. The revised Atlanta classification of acute pancreatitis was used to assess: (1) abdominal pain (NRS $>4$ ) $>24$ hours after drainage; (2) amylase level more than three times normal level; and (3) gastrointestinal perforation, acute cholecystitis or acute peritonitis [11]. Clinical diagnosis of hemobilia consisted of: (1) blood mixed with bile after biliary drainage; (2) slight bleeding indicated by a small amount of black stool or positive occult blood test; and (3) moderate to severe bleeding, indicated by a drop in hemoglobin of $3 \mathrm{~g} / \mathrm{L}$, requiring blood transfusion, angiographic intervention, or surgery $[12,13]$. Successful drainage was defined as a reduction in serum bilirubin level to < $50 \%$ of the pre-drainage value within 2 weeks or normalization after the procedure without any complications during the follow-up period [14].

\section{Statistical method}

All statistical analyses were performed with Stata (version 12.0) statistical software. Continuous variable data were reported as mean \pm SD for parametric data and median with interquartile ranges (IQR) for nonparametric data or as counts and percentages for categorical variables. Continuous variables were also expressed as ranges. We also compared baseline characteristics and post-drainage complications. Data were analyzed by t-test, and the $X 2$ test was used to investigate qualitative variables. Results were presented as relative risk (RR) with $95 \%$ confidence intervals (Cls), and $P<0.05$ was considered statistically significant.

Reported incidence of cholangitis after biliary drainage varied from $22 \%$ to $46 \%$ [12]. Our prior experience showed a cholangitis rate of $40 \%$. To detect a difference with a significance level $(\alpha)$ of 0.05 and a power of $80 \%$ with a two-tailed test, and to account for a $10 \%$ loss to follow-up, it was estimated that 150 patients were needed for the study. Therefore, we enrolled a total of 180 patients.

\section{Results}

\section{Patient characteristics}

A total of 180 patients were selected for the study, 99 patients received ERCP, and 81 patients underwent PTBD. There were no significant differences in patient demographics, including age, sex, and Bismuth classification distribution. No significant differences were noted in WBC count, TBIL, or tumor marker CA19 -9 between the two groups $(P>0.05)$ ( $\triangleright$ Table 1$)$.

\section{Impact of ERCP and PTBD drainage on serum total bilirubin}

Of the 99 patients in the ERCP group, 34 underwent endoscopic unilateral drainage with a single biliary stent, 46 patients had bilateral biliary stent placement, and another 19 patients received nasobiliary drainage. Forty-nine of the 81 patients in
- Table 1 Baseline characteristics.

\begin{tabular}{|c|c|c|c|}
\hline & $\begin{array}{l}\text { ERCP group } \\
(n=99)\end{array}$ & $\begin{array}{l}\text { PTBD group } \\
(n=81)\end{array}$ & $P$ \\
\hline \multicolumn{4}{|l|}{ Age (y) } \\
\hline$<65$ & 26 & 32 & \multirow[t]{3}{*}{0.13} \\
\hline $65-75$ & 48 & 29 & \\
\hline $75>$ & 25 & 20 & \\
\hline \multicolumn{4}{|l|}{ Sex } \\
\hline Male & 55 & 43 & \multirow[t]{2}{*}{0.74} \\
\hline Female & 44 & 38 & \\
\hline \multicolumn{4}{|l|}{ Types } \\
\hline II & 28 & 20 & \multirow[t]{3}{*}{0.75} \\
\hline III & 27 & 26 & \\
\hline IV & 44 & 35 & \\
\hline $\begin{array}{l}\mathrm{WBC}\left(\times 10^{2} / \mathrm{L}\right) \\
(\text { mean } \pm \mathrm{SD})\end{array}$ & $7.33 \pm 2.81$ & $7.91 \pm 3.60$ & 0.343 \\
\hline $\begin{array}{l}\text { TBIL (umol/L) } \\
(\text { mean } \pm \text { SD) }\end{array}$ & $\begin{array}{l}303.17 \pm \\
182.72\end{array}$ & $\begin{array}{l}332.14 \pm \\
186.85\end{array}$ & 0.242 \\
\hline $\begin{array}{l}\text { CA19-9 (kU/L) } \\
(\text { mean } \pm \text { SD) }\end{array}$ & $\begin{array}{l}700.52 \pm \\
457.87\end{array}$ & $\begin{array}{l}681.34 \pm \\
405.27\end{array}$ & 0.769 \\
\hline $\begin{array}{l}\text { Hospitalization time } \\
(\text { days })(\text { mean } \pm S D)\end{array}$ & $14.32 \pm 7.84$ & $14.86 \pm 9.19$ & 0.671 \\
\hline $\begin{array}{l}\text { Hospitalization costs } \\
(\mathrm{RMB})(\text { mean } \pm \text { SD })\end{array}$ & $\begin{array}{l}44406.67 \pm \\
12407.3\end{array}$ & $\begin{array}{l}22300.5 \pm \\
8567.9\end{array}$ & 0.005 \\
\hline
\end{tabular}

ERCP, endoscopic retrograde cholangiopancreatography; PTBD, percutaneous transhepatic biliary drainage; WBC, white blood cell; TBIL, total bilirubin; SD, standard deviation; RMB, renminbi

- Table 2 Effects of different drainage methods on serum total bilirubin.

\begin{tabular}{|c|c|c|}
\hline & TBIL (umol/L) & $P$ \\
\hline \multicolumn{3}{|l|}{ ERCP group $(n=99)$} \\
\hline - Pre-drainage (mean $\pm S D$ ) & $303.17 \pm 182.72$ & \multirow[t]{2}{*}{0.001} \\
\hline - Post-drainage (mean $\pm S D)$ & $126.87 \pm 75.96$ & \\
\hline \multicolumn{3}{|l|}{ PTBD group $(n=81)$} \\
\hline - Pre-drainage (mean $\pm S D)$ & $332.14 \pm 186.85$ & \multirow[t]{2}{*}{0.001} \\
\hline - Post-drainage (mean $\pm S D$ ) & $161.42 \pm 93.11$ & \\
\hline $\begin{array}{l}\text { ERCP group for decreasing of TBIL } \\
(\text { mean } \pm S D)\end{array}$ & $176.30 \pm 106.76$ & \multirow[t]{2}{*}{0.810} \\
\hline $\begin{array}{l}\text { PTBD group of decreasing of TBIL } \\
(\text { mean } \pm \text { SD) }\end{array}$ & $170.72 \pm 93.74$ & \\
\hline $\begin{array}{l}\text { TBIL, total bilirubin; ERCP, endoscopic } \\
\text { phy; PTBD, percutaneous transhepatic } \\
\text { tion }\end{array}$ & $\begin{array}{l}\text { grade cholangiopan } \\
\text { ary drainage; SD, star }\end{array}$ & $\begin{array}{l}\text { ogra- } \\
\text { devia- }\end{array}$ \\
\hline
\end{tabular}


the PTBD group had external drainage with the catheter tip left above the obstruction and 32 had internal-external drainage with the tip of the catheter in the duodenum. Compared with pre-drainage, total bilirubin decreased significantly both in the ERCP group $(303.17 \pm 182.72$ vs. $126.87 \pm 75.96, P=$ $0.001)$ and the PTBD group $(332.14 \pm 186.85$ vs. $161.42 \pm$ 93.11, $P=0.001$ ) after 2 weeks of drainage. But there was no statistical difference in decrease in total bilirubin between the two groups (176.30 \pm 106.76 in ERCP group vs. $170.72 \pm 93.74$ in PTBD group, $P=0.81$ ) ( $\triangleright$ Table 2 ).

\section{Complications}

Fifty-five of 180 patients (30.56\%) developed post-drainage cholangitis: 37 in the ERCP group and 18 in the PTBD group. Among them, 46 cases (83.64\%) of post-procedure acute cholangitis were considered as bacterial contamination originating from the duodenum, eight cases (14.55\%) with drainage tube migration and 1 case $(1.81 \%)$ with blockage of drainage tube caused by hemobilia. Most fevers occurred within 24 hours after intervention.

Incidence of cholangitis was significantly higher in the ERCP group than in the PTBD group ( $37.37 \%$ vs. $22.22 \%, P=0.028)$. Subgroup analysis showed similar results between the two groups when comparing patients with types III and IV hilar obstruction (36.62\% in ERCP group vs. $18.03 \%$ in PTBD group, $P=$ $0.018)$. There was no significant difference in patients with type II obstruction between the two groups (39.29\% in ERCP group vs. $35.00 \%$ in PTBD group, $P=0.762$ ). Four of 180 patients $(2.22 \%)$ had hemobilia, and there was no statistical difference between the two groups $(2.02 \%$ in ERCP group vs. $2.47 \%$ in
PTBD group, $P=0.76$ ). Nineteen of 180 patients (10.56\%) developed post-procedure pancreatitis, with significantly more patients in the ERCP group than in the PTBD group (17 [17.17\%] vs. 2 [2.47\%], $P=0.001)$. Twenty-three of 180 (12.78\%) patients required salvage biliary drainage, again with significantly more patients in the ERCP group than in the PTBD (18 [18.18\%] vs. 5 [6.17\%], $P=0.029$ ) ( Fig.3). The type II HC patients who received unilateral stent placement had the lowest rate of postdrainage cholangitis and types III or IV HC patients with PTBD external drainage had the lowest incidence of cholangitis (> Fig.4).

\section{Incidence of cholangitis in various subgroups}

In the ERCP group, patients with endoscopic bilateral biliary stent placement had a higher rate of post-drainage cholangitis than those who received unilateral biliary stent placement ( $50.00 \%$ vs. $26.47 \%, P=0.034$ ). There was no significant difference between those who had endoscopic unilateral stenting and those who had endoscopic nasobiliary drainage (ENBD) (26.47\% vs. $26.32 \%, P=0.99$ ). For the PTBD group, patients who underwent PTBD internal-external drainage had a higher rate of cholangitis than those with only external drainage (34.36\% vs. $14.29 \%, P=0.034$ ) ( Fig. 3 ).

\section{Discussion}

Preoperative biliary drainage plays a positive role in enhancing surgical safety for patients with types II, III, and IV potentially resectable hilar cholangiocarcinoma with jaundice. This method not only improves retention of liver function after radical

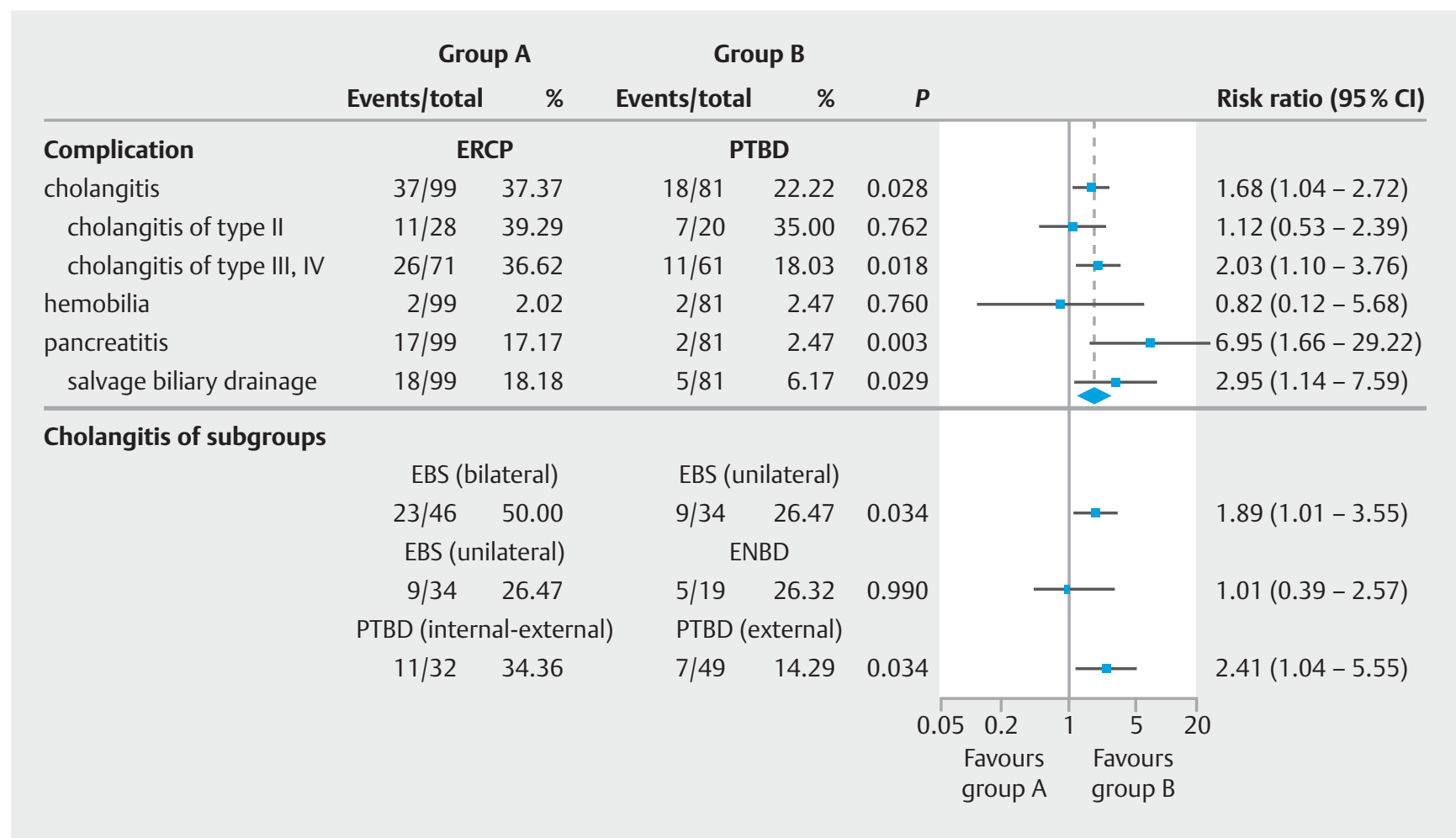

Fig. 3 Incidence of complications with various types of drainage. 


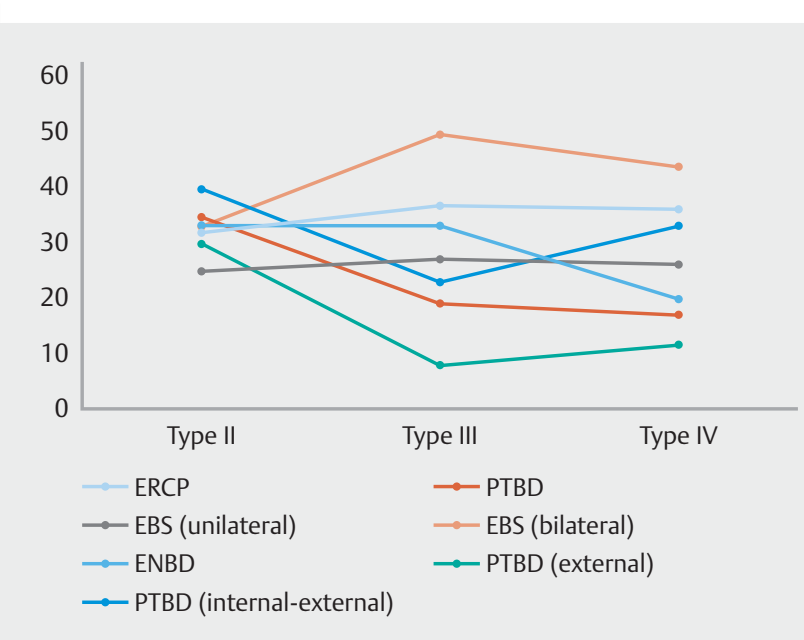

- Fig. 4 The tendency of post-drainage cholangitis after ERCP/ PTBD in types II, III and IV HC.

surgery, but also speeds regeneration of the remnant liver [15]. It also should be noted that not all patients can benefit from preoperative biliary drainage, for instance, those with drainage-associated cholangitis, a critical predictor that can result in liver insufficiency and liver failure and could even increase the chance of mortality after radical surgery [16-18]. Currently, there is no consensus about how preoperative biliary drainage should be performed worldwide. Kloek JJ et al [19] found that preoperative PTBD could outperform EBS in patients with resectable $\mathrm{HC}$, because PTBD was associated with fewer infectious complications and involved fewer procedures. A Japanese clinical practical guideline [20], however, recommended ERCP as the first choice for preoperative drainage of biliary tract cancers. It is important to note that the guideline was aimed at all biliary tract cancers rather than HC. How to define the optimal method is still debated.

The basis of an ideal drainage plan is maximization of drainage volume of the expected retention liver and minimization of drainage-associated cholangitis at the same time [21]. To drain bile from the future remnant lobe adequately, bilateral plastic stents (t2 stents) were inserted in the bile duct of the retained liver during our ERCP operations. Vienne et al reported that they needed double-sided stent insertion if more than $50 \%$ of the liver drainage volume was expected, but our results showed that single-sided stent insertion could also produce a good jaundice reduction effect [22]. Both the double-sided stent insertion group and the single-sided stent insertion group with types III and IV potentially resectable hilar cholangiocarcinoma exhibited obvious bilirubin number reduction and similar levels of bilirubin drop $(P=0.01)$. Besides this, jaundice reduction effects were also similar in the ERCP and PTBD groups and in their corresponding subgroups $(P>0.05)$.

The US consensus statement on hilar cholangiocarcinoma published in 2013 suggested that PTBD is superior in reducing risks of complications [23]. We recently published results of a meta-analysis that testify to PTBD as the preferred initial drainage method for patients with types II, III, and IV tumor obstruc- tion [24]. Many centers also reported a higher incidence of acute cholangitis after ERCP. Min et al. reported 106 cases of hilar cholangiocarcinoma, with 44 undergoing ERCP versus 62 with PTBD. They found higher rates of cholangitis in the ERCP group than in the PTBD group $(54.5 \%$ vs. $22.6 \%, P<0.05)$ [25]. In our research, we found that incidence of post-drainage cholangitis, pancreatitis, and secondary remedial biliary drainage were greater in the ERCP group than in the PTBD group $(P<$ 0.05). We analyzed the relationship between post-drainage cholangitis and Bismuth-Corlette classification and found that type II HC patients showed no difference in incidence of postdrainage cholangitis (39.29\% vs. $35.00 \%, P=0.762$ ), only with types III and IV HC was incidence of post-drainage cholangitis higher in the ERCP group than in the PTBD group $(36.62 \%$ vs. $18.03 \%, P=0.018)$.

Choice of endoscopic unilateral stenting versus bilateral stenting remains controversial. De Palma found that rates of cholangitis were higher with bilateral stenting than with unilateral stenting $(16.6 \%$ vs. $8.8 \%, P<0.05)$ [26]. Iwano reported that liver abscesses occurred more frequently in the bilateral stenting group than in the unilateral stenting group $(17.6 \%$ vs. $1.5 \%, P<0.05)$ [27]. In our study, incidence of cholangitis was significantly higher among patients with endoscopic bilateral drainage than in those with endoscopic unilateral drainage (50.00\% vs. 26.47\%, $P=0.034$ ). Considering patients with types II, III, and IV potentially resectable HC whose operative treatment should be performed soon after the diagnosis is ascertained, endoscopic unilateral stenting and endoscopic bilateral biliary stents were no different in drainage effect. Besides, incidence of post-drainage cholangitis from endoscopic bilateral biliary stent insertion was higher, so there were more advantages to unilateral endoscopic biliary stenting for radical resection of hilar cholangiocarcinoma, particularly in type II or III cases.

Some endoscopists have advocated use of ENBD for preoperative biliary drainage for hilar cholangiocarcinoma. Kawakami reported that cholangitis secondary to stent occlusion was significantly more common in the stenting group than in the ENBD group $(60.0 \%$ vs. $10.0 \%, P<0.05)$. The nasobiliary catheter may have negative pressure, making the bile flow more easily [28]. However, we observed that the cholangitis rate was comparable between the group with endoscopic unilateral stenting insertion and the ENBD group $(26.47 \%$ vs. $26.32 \%, P=$ $0.99)$. ENBD has the disadvantage of causing nose discomfort and is at risk of dislocation. Because external bile loss leads to water-electrolyte imbalance, radical resection of hilar tumor is recommended within 4 weeks of ENBD placement. An alternative option to provide internal drainage is cutting the ENBD tube, using a pair of endoscopic scissors, and leaving the tip of the drain in the stomach to minimize risk of external bile loss.

In patients with PTBD, internal drainage is achieved by leaving the tip of the drain catheter in the duodenum. Internal drainage minimizes external bile loss and maintains water-electrolyte balance, which also improves nutritional status [29]. In our study, incidence of cholangitis in those with external/internal drainage was higher than in those with external drainage alone $(34.36 \%$ vs. $14.29 \%, P=0.034)$. We hypothesized that 
the difference in incidence of cholangitis was secondary to reflux of duodenal content into the biliary system through the catheter when the tip is placed in the duodenum. Comparing patients with endoscopic single stent placement, ENBD, and those with external drainage alone in the PTBD group, incidence of cholangitis was $26.47 \%, 26.32 \%$, and $14.29 \%$, respectively. Although endoscopic unilateral stenting insertion and ENBD can reduce incidence of cholangitis, PTBD with external drainage resulted in the lowest incidence of cholangitis. PTBD with external drainage alone may be the preferred drainage method, especially for salvage biliary drainage when endoscopic stenting fails.

Many aspects of preoperative biliary drainage need to be considered for patients with types II, III, and IV HC. Our results appeared to show that PTBD external drainage for types III and IV HC has more advantages. Individualized experience with drainage operation is also a necessity, and the competency of the hospital's biliary intervention or endoscopic techniques should be considered comprehensively. The study also has some limitations. It was single-center and retrospective with a relatively small number of patients. Data on long-term clinical outcomes, including stent patency and patient surgical mortality during follow-up are limited. A prospective, randomized controlled trial with a larger number of patients is needed.

\section{Conclusion}

In conclusion, percutaneous transhepatic biliary external drainage may be the preferred preoperative drainage method for hilar cholangiocarcinoma because of its low incidence of cholangitis and pancreatitis. If a patient is not suitable for PTBD, unilateral EBS or ENBD can be used as a secondary option.

\section{Acknowledgements}

This work was supported by the Free Exploration Project of the Central Universities, Lanzhou University (No. 18LZUJBWZY054) and Teaching Research Project of Lanzhou University (No. 2017218). Clinical trial registration: NCT03104582

\section{Competing interests}

None

\section{References}

[1] Polistina FA, Guglielmi R, Baiocchi C et al. Chemoradiation treatment with gemcitabine plus stereotactic body radiotherapy for unresectable, non-metastatic, locally advanced hilar cholangiocarcinoma. Results of a five year experience. Radiother Oncol 2011; 99: 120-123

[2] Paik WH, Park YS, Hwang JH et al. Palliative treatment with self-expandable metallic stents in patients with advanced type III or IV hilar cholangiocarcinoma: a percutaneous versus endoscopic approach. Gastrointest Endosc 2009; 69: 55-62

[3] Byrnes V, Afdhal N. Cholangiocarcinoma of the hepatic hilum (Klatskin tumor). Curr Treat Options Gastroenterol 2002; 5: 87-94
[4] Castellano-Megías VM, Andrés ID, Colina-Ruizdelgado F. Pathological aspects of so called "hilar cholangiocarcinoma". World J Gastrointest Oncol 2013; 5: 159-170

[5] Ramos E. Principles of surgical resection in hilar cholangiocarcinoma. World J Gastrointest Oncol 2013; 5: 139-146

[6] Govil S, Reddy MS, Rela M. Surgical resection techniques for locally advanced hilar cholangiocarcinoma. Langenbeck Arch Surg 2014; 399: $707-716$

[7] Rd VV, Cosgrove D, Herman JM et al. Management of perihilar cholangiocarcinoma in the era of multimodal therapy. Expert Rev Gastroent 2012; 6: $481-495$

[8] Soares KC, Kamel I, Cosgrove DP et al. Hilar cholangiocarcinoma: diagnosis, treatment options, and management. Hepatobil Surg Nutr 2014; 3: $18-34$

[9] Spiegel B, Bolus R, Harris LA et al. Measuring irritable bowel syndrome patient-reported outcomes with an abdominal pain numeric rating scale. Aliment pharm therap 2009; 30: 1159-1170

[10] Kiriyama S, Takada T, Strasberg SM et al. TG13 guidelines for diagnosis and severity grading of acute cholangitis (with videos). J Hepatobiliary Pancreat Sci 2013; 20: 24-34

[11] Banks PA, Bollen TL, Dervenis C et al. Classification of acute pancreatitis-2012: revision of the Atlanta classification and definitions by international consensus. Gut 2013; 62: 102-111

[12] Cotton PB, Garrow DA, Gallagher J et al. Risk factors for complications after ERCP: a multivariate analysis of 11,497 procedures over 12 years. Gastrointest Endosc 2009; 70: $80-88$

[13] Parikh K, Ali MA, Wong RC. Unusual causes of upper gastrointestinal bleeding. Gastrointest Endosc Clin N Am 2015; 25: 583-605

[14] Aljiffry M, Abdulelah A, Walsh M. Evidence-based approach to cholangiocarcinoma: a systematic review of the current literature. J Am Coll Surg 2009; 208: $134-147$

[15] Farges O, Regimbeau JM, Fuks D et al. Multicentre European study of preoperative biliary drainage for hilar cholangiocarcinoma. Br J Surgery 2013; 100: $274-283$

[16] Lafemina J, Jarnagin WR. Surgical management of proximal bile duct cancers. Langenbeck Arch Surg 2012; 397: 869-879

[17] Sakata J, Shirai Y, Tsuchiya Y et al. Preoperative cholangitis independently increases in-hospital mortality after combined major hepatic and bile duct resection for hilar cholangiocarcinoma. Langenbeck Arch Surg 2009; 394: 1065 - 1072

[18] Ribero D, Zimmitti G, Aloia TA et al. Preoperative cholangitis and future liver remnant volume determine the risk of liver failure in patients undergoing resection for hilar cholangiocarcinoma. J Am Coll Surg 2016; 223: 87-97

[19] Kloek J], Na VDG, Aziz Y et al. Endoscopic and percutaneous preoperative biliary drainage in patients with suspected hilar cholangiocarcinoma. J Gastrointest Surg 2010; 14: 119-125

[20] Miyazaki M, Yoshitomi H, Miyakawa S et al. Clinical practice guidelines for the management of biliary tract cancers 2015: the 2nd English edition. J Hepatobiliary Pancreat Sci 2015; 22: 249-273

[21] Rerknimitr R, Angsuwatcharakon P, Ratanachu-Ek T et al. Asia-Pacific consensus recommendations for endoscopic and interventional management of hilar cholangiocarcinoma. J Gastroenterol 2013; 28 : $593-607$

[22] Vienne A, Hobeika E, Gouya $\mathrm{H}$ et al. Prediction of drainage effectiveness during endoscopic stenting of malignant hilar strictures: the role of liver volume assessment. Gastrointest Endosc 2010; 72: 728 - 735

[23] Yasuda I, Mukai T, Moriwaki H. Unilateral versus bilateral endoscopic biliary stenting for malignant hilar biliary strictures. Digest Endosc 2013; 25: $81-85$

[24] Tang Z, Yang Y, Meng W et al. Best option for preoperative biliary drainage in Klatskin tumor: A systematic review and meta-analysis. Medicine 2017; 96: e8372 
[25] Min KK, Won PJ, Kyun L] et al. A comparison of preoperative biliary drainage methods for perihilar cholangiocarcinoma: endoscopic versus percutaneous transhepatic biliary drainage. Gut Liver 2015; 9: $791-799$

[26] De Palma GD, Galloro G, Siciliano S et al. Unilateral versus bilateral endoscopic hepatic duct drainage in patients with malignant hilar biliary obstruction: results of a prospective, randomized, and controlled study. Gastrointest Endosc 2001; 53: 547 - 553

[27] Iwano H, Ryozawa S, Ishigaki N et al. Unilateral versus bilateral drainage using self-expandable metallic stent for unresectable hilar biliary obstruction. Digest Endosc 2011; 23: 43-48
[28] Kawakami H, Kuwatani M, Onodera M et al. Endoscopic nasobiliary drainage is the most suitable preoperative biliary drainage method in the management of patients with hilar cholangiocarcinoma. J Gastroenterol 2011; 46: 242-248

[29] Xu C, Huang XE, Wang SX et al. Comparison of infection between internal-external and external percutaneous transhepatic biliary drainage in treating patients with malignant obstructive jaundice. Asian Pac J Cancer Prev 2015; 16: 2543-2546 